\title{
Study on Artificial Intelligence Controller Based on Single-chip Microcomputer
}

\author{
Yong Shi ${ }^{1, ~ a}$, Wei Wang ${ }^{2, ~ b}$, Chong $\mathrm{Xu}^{3, \mathrm{c}}$,Ning Tao ${ }^{4, \mathrm{~d}}$ and Xin Liu ${ }^{5 \mathrm{e}}$ \\ ${ }^{1}$ BIM Computing Research Center, Shenyang jianzhu University, Shengyang, 110168, China \\ 2 BIM Computing Research Center, Shenyang jianzhu University, Shengyang, 110168, China \\ ${ }^{3}$ BIM Computing Research Center, Shenyang jianzhu University, Shengyang, 110168, China \\ ${ }^{4}$ BIM Computing Research Crnter, Shenyang jianzhu University, Shengyang, 110168, China \\ ${ }^{5}$ BIM Computing Research Crnter, Shenyang jianzhu University, Shengyang, 110168, China \\ a Shiyong13@163.com, b519836646@qq.com,32500103@ qq.com, d23010150@qq.com, \\ '6620274@qq.com
}

Keywords: AT89S52; PID control; Human-simulated intelligent control; Sensors

\begin{abstract}
This article take the boiler water temperature controller for research object and investigate the control system based on taking the SCM a microcontroller as the core as a boiler controller, sensor technology, the basis for the system design to achieve the temperature and water level of the collection. AT89S52 microcontroller as the core of the system, combined with simulated intelligent PID control algorithm performance analysis, and hardware design, but also for the actual boiler research to meet the energy saving boiler control and safety requirements.
\end{abstract}

\section{Introduction}

Heating boiler drum water level and water temperature protection control in the whole system plays a very important role in the central heating boiler drum run, maintaining water level and water temperature within a certain range to ensure the necessary conditions for the normal operation of the boiler ${ }^{[1,2]}$. Water protection function should satisfy the time when the boiler water protection from Ganguo and burn water wall ${ }^{[3,4]}$, when there is full of water valve opens automatically, when the water level reached the limits of water level will shutdown, shut down, turn off the main Valve to prevent equipment damage ${ }^{[5,6]}$.

\section{Circuit Design of the Boiler Master Controller}

\section{A. Temperature Acquisition Circuit}

Temperature Acquisition using the DS18B20 digital temperature sensor, the sensor if required it can be accurate temperature conversion, I / O line must ensure power supply during the conversion. Since the work of current to $1 \mathrm{~mA}$ of DS18B20, so the pull-up resistor $5 \mathrm{~K}$ alone is not enough to provide power, and when there are a few DS18B20 hanging in the same I / O lines and also to the Way is to DS18B20 supply VDD pin is access from an external power supply, shown in temperature conversion, the problem is more acute. Figure 1. The advantage is I / O line does not need to strengthen the pull, and bus controller not always remain high during the conversion temperature. This can allow the conversion during the single data bus for other exchanges. In addition, single bus can carry as many pieces DS18B20 temperature sensor, and if they all use external power, then it can first send a Skip ROM command, followed by a Convert T command, so that they at the same time the temperature conversion. Note that when coupled with an external power supply, GND pins can not be left floating. When the temperature is higher than $100{ }^{\circ} \mathrm{C}$, the parasitic power use is not recommended, because in this temperature DS18B20 show relatively large leakage current, communications may not be possible. In this case the temperature should be used DS18B20 the VDD pin. 


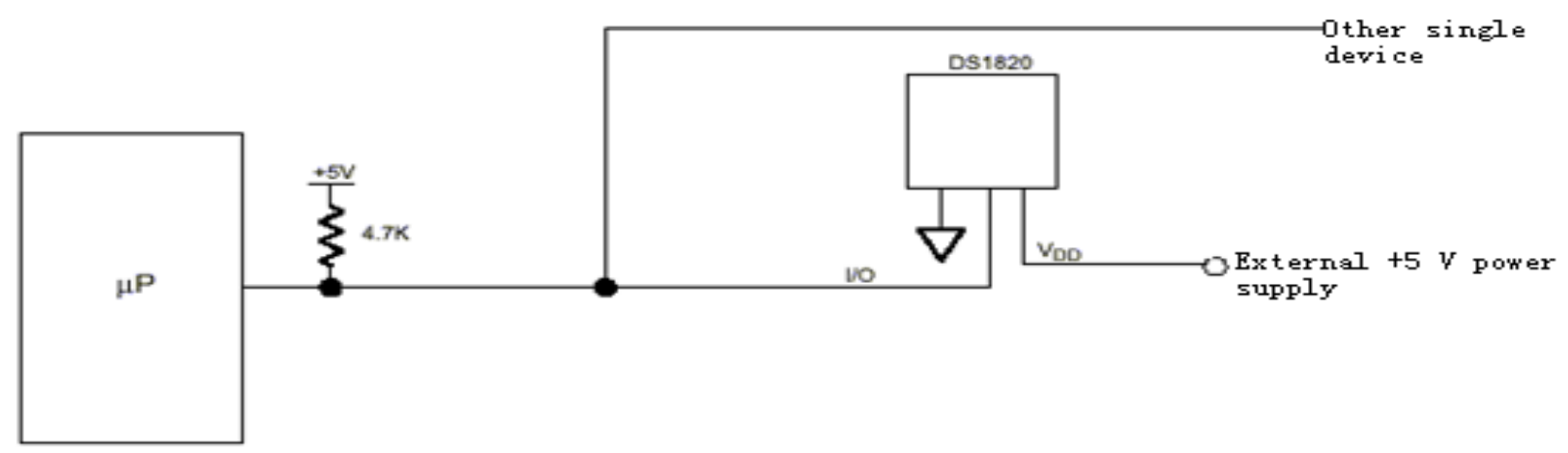

Figure 1. Reference to an external power supply

\section{B. Water Level Monitoring Circuit}

Hall liquid level sensor installed in the container outside the permanent magnet in the float on the branch, with the level changes, the role of the Hall devices on the changes in the magnetic flux density, which measured level. Structure shown in Figure 2.

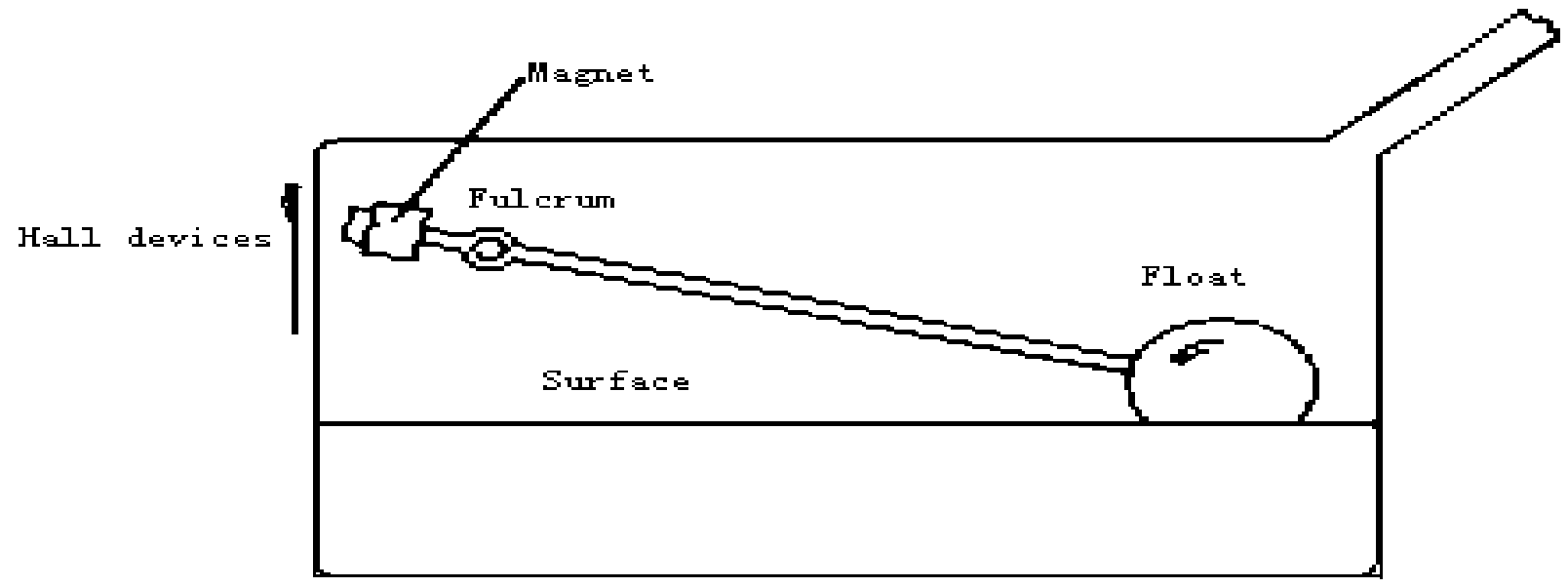

Figure 2. Hall devices measuring the water level

Hall effect sensor is achieved by Hall converted a magnetic sensor. It has high sensitivity and wide application characteristics. Its working principle is: a semiconductor thin, its length L, width B, thickness $\mathrm{D}$, magnetic induction placed in the magnetic field strength $\mathrm{B}$, in opposite sides of the pass to control the current I, and orthogonal magnetic field direction and current direction, Then both sides will produce a semiconductor with the control current and the magnetic induction is proportional to the product of the electric potential $U$, is the Hall voltage of the electric potential, with the $\mathrm{U}_{\mathrm{H}}$, said that $\mathrm{U}_{\mathrm{H}}=\mathrm{K}_{\mathrm{H}} \mathrm{IB}$, where $\mathrm{K}_{\mathrm{H}}$ is the sensitivity of the Hall element, the semiconductor thin Is the Hall element

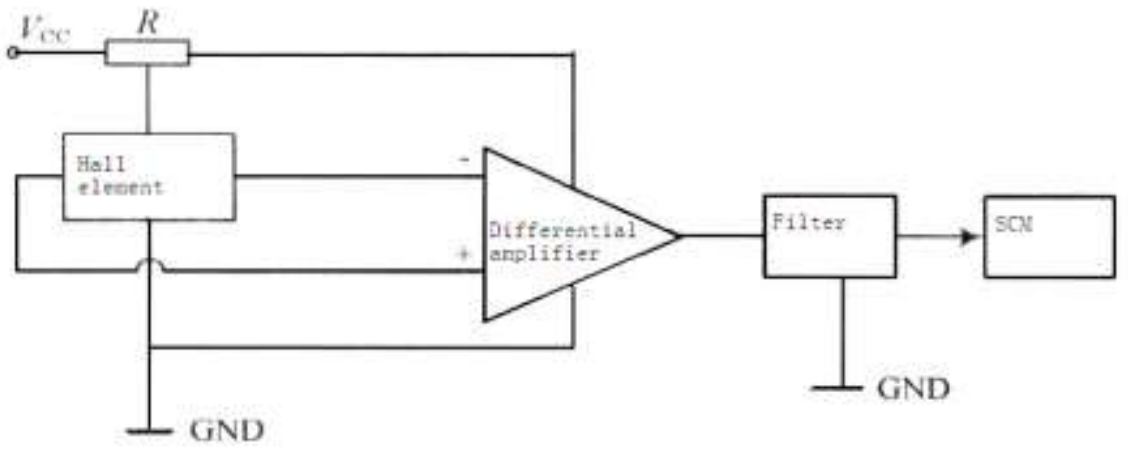

Figure 3. Hall element connected with the microcontroller 
When the surface area far greater than the distance between the two when the magnetic field strength of the middle $\mathrm{O}$, along the $\mathrm{z}$ axis in the gap between the formation of a uniform magnetic field gradient $\mathrm{dB} / \mathrm{dx}=\mathrm{K}$ ( $\mathrm{K}$ is a constant.) $\mathrm{B}=0$ Service as a reference to the origin of the displacement $\mathrm{x}$, then $\mathrm{x}=\mathrm{O}$ when, $\mathrm{B}=\mathrm{O}, \mathrm{U}_{\mathrm{H}}=\mathrm{O}$. When they move to the middle of the Hall element $\mathrm{x}$ at the time, $\mathrm{U}_{\mathrm{H}}$ Department of $\mathrm{B}$ size determined by the $\mathrm{x} . \mathrm{U}_{\mathrm{H}}=\mathrm{K}_{\mathrm{H}} \mathrm{IB}$ known by the formula: I remain unchanged, $\mathrm{dU}_{\mathrm{H}} / \mathrm{dx}=\mathrm{IK}_{\mathrm{H}} \mathrm{dB} / \mathrm{dx}=\mathrm{K}_{\mathrm{H}} \mathrm{I}=\mathrm{K}$, points after that $\mathrm{U}_{\mathrm{H}}=\mathrm{Kx}$, the Hall potential is proportional to the displacement. The greater the magnetic field gradient, the higher the sensitivity, the more uniform magnetic field, $\mathrm{U}_{\mathrm{H}}$, and $\mathrm{x}$ the linear as possible. The connection diagram of the Hall element and the microcontroller is shown in Figure 3.

Hall element with the induced magnetic field intensity voltage signals, signal amplification by the differential amplifier, and then after a filter is filtering to get a clearer signal, the signal again after A / D converter to convert the spread to the microcontroller for processing. The design is by the microcontroller as a master controller, microcontroller to control the thyristor heating, lower machine PID regulator, to the signal converter and solenoid valve, add water by adjusting the inverter control pump and control solenoid valve

\section{Human-simulated Intelligent PID Control Algorithm and the Capability Analysis of Control- ling Algorithm}

Human-simulated intelligent PID control combines the traditional PID control algorithm and human-simulated intelligent control algorithm together and uses proportion, integral and differential control function to realize selecting feature information and combined control scheme according to dynamic process. In human-simulated intelligent control, the solving information space is made up of system error e $(t)$, error rate e' $(t)$ and time t. e $(t)=r(t)-y(t), r(t)$ is input, $y(t)$ is output. Apparently whether fixed value control or servo control, the aim is when $t$ tends to infinity, e $(t)=e^{\prime}(t)$ $=0$. The movement trajectory of system in the information space not only reflects all dynamic information but also reflects the control function in the system. It can be seen from the static characteristics, the system deviation from the equilibrium state, the error increases when it is the direction of proportional control, error reaches extreme point, the control volume reaches the maximum, then decreases the error began, Control the amount of time the most extreme point with a lot more than a small amount of error and no longer change with the change, but "wait ", "observation" error changes. When the error is zero when the system has the opposite direction to increase the percentage of control. Have roughly the same repetition and control process, so that after one or two oscillations, the system quickly stabilize the new equilibrium point.

From the automatic control system performance indexes, the concrete analysis of the humansimulated intelligent PID is as the followings:

The Stability The method of combining open-loop and closed-loop is good for stability of large delay system. From energy perspective, when the error increases, human-simulated intelligent PID controller inputs larger control energy by using the proportional control to offset disturbance input energy. When the error decreases, the intelligent control algorithm switches to the open-loop holding mode to guard its change. Such strategy avoids system oscillation and improves the system stability effectively.

Quickness For multi-model control, because the system will automatically switch control mode, so when the error becomes larger or has increasing trend, it can input larger control quantity and the system reflects quickly. In addition, the system can stabilize after one switch mode by setting appropriate parameters and it also shortens the setting time.

Accuracy Because of using the open-loop hold mode and control depending on memory characteristic, multi-model control have integration function and the system stable error is zero. Overall, relying on the flexible recognition and decision control method, the multi-model control mode solves the problem of the control quality (power, stability, accuracy) in contradiction.

AT89S52 microcontroller programming, PID control program flow chart in Figure 4 


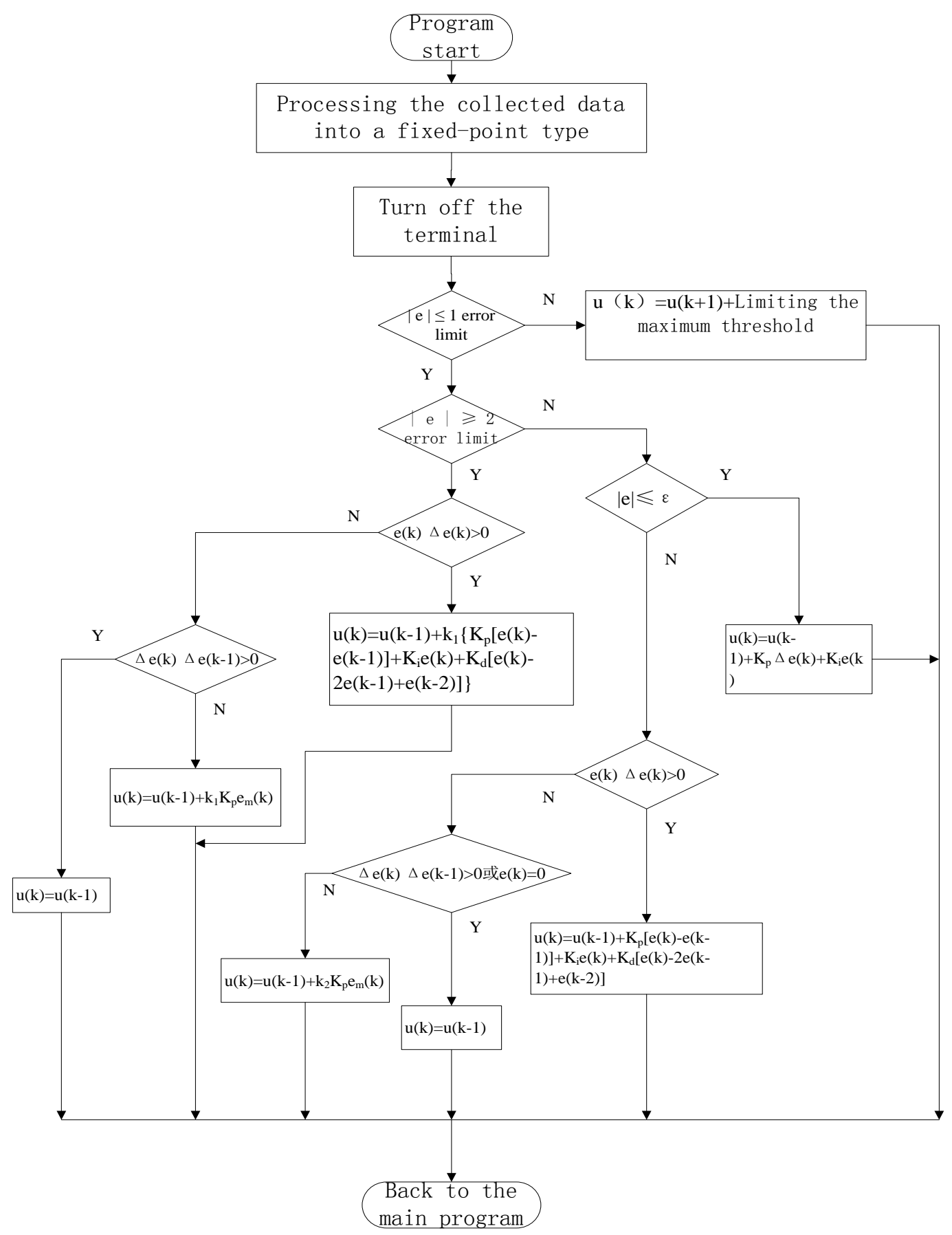

Figure 4. PID control program flow chart

\section{Conclusion}

In this paper, SCM boiler control system, combined with practical requirements of the boiler control operation designed to SCM (AT89S52) control the main line, with simulated intelligent PID control design can be achieved out of the boiler water level, temperature control of the hardware circuit, the system SCM control system with a series of advantages, less selection, and simple circuit structure, software function quite perfect, cost-effective. Boilers can control some of the more simple, with a certain value. 


\section{References}

[1] Lanjun Li, Shouyi Yu, Song GAO. Fuzzy PID Control of Micro-Boiler Level [J]. Computer ,2007,11-1:33

[2] Songyue Peng, Liu fruit. PLC based control system of heating furnace temperature [J]. Automation and Applications, 2007,10:32

[3] Ai-Ghazzawi A,Ali E, Nouh A, et al,On-line tuning strategy for model predictive controllers [M],Journal of Process Control,2001

[4] Mansour S E, Kember G C, Dubay R, et al. Onlineoptimization of fuzzy2PID control of a thermal process[J].ISA Transactions,2005,44 (4):305 - 314.

[5] Liping Zhang, Guili Peng. PLC-based central heating boiler feed water protection control, 2004 : pp.3 6.

[6] Mingli,Guangzheng Peng. Fuzzy PID control algorithm in cylinder position servo control application.J.Machine and hydraulic ,2004,10 